\title{
M-RPL: A Design Algorithm for WSNs with Mixed Traffic
}

\author{
Annop Monsakul
}

\begin{abstract}
A design of wireless sensor network to support traffic engineering for both unicast and multicast traffic is a very difficult problem. This paper proposes a design algorithm called M-RPL. It is IPv6 based routing protocol for low power, lossy Networks (LLNs) that concern routing of both types of traffic. However, since multicast traffic model could be employed in many situations and could be managed by various kinds of multicast routing protocols. The efficiency of M-RPL is evaluated for various traffic demands and networks of 100 node and the total unicast traffic in/out per node of 64, 128, 256, 512 and 1024 kbps, compared with RPL. The experimental results show that, in almost all cases, M-RPL give better performance in term of installation cost.
\end{abstract}

Index Terms - Traffic engineering, wireless sensor network, IPv6 routing protocol, spanning tree.

\section{INTRODUCTION}

Nowadays, a wireless sensor network (WSNs) are very popular technology, it is a wireless network consisting of spatially distributed autonomous devices using sensors to environmental conditions. Commonly monitored parameters are temperature, humidity, pressure, wind speed, sound intensity, power-line voltage, vital body functions, etc. WSN [1] appears as an essential platform for prominent concept of Internet of Things (IoT).

IPv6 Routing Protocol for Low power and Lossy Networks (RPL) is the standard IPv6 based routing protocol for low power, lossy Networks (LLNs) consist largely of constrained nodes with limited power, memory, and processing resources as proposed by IETF such as WSNs. It is a routing protocol [2] adapted for information routing with low power, low storage and processing sensor devices. RPL are supports traffic flows include Multipoint-to-Point (MP2P), Point-to-Multipoint (P2MP), and Point-to-Point (P2P) traffic. Thus, IP version 6 (IPv6) is an answer to solve this problem or IPv6 called Internet Protocol Next Generation (IPng), as IPv6 has 128-bit address space pushing the theoretical limit unique IPv6 nodes to roughly 3.4E1038 unique addresses, which is more enough for new devices in the future. The first time Internet and Internet Protocol (IP) were designed to provide a best-effort, fair delivery service. Under a best-efforts scheme, Internet treats all packets equally, no guarantees, no special resources

Manuscript received March 20, 2016; revised May 19, 2016.

Annop Monsakul is with the Faculty of Information of Technology, Thai-Nichi Institute of Technology, 1771/1, Pattanakarn Rd, Suan Luang, Bangkok, 10250, Thailand (e-mail: annop@tni.ac.th). allocation; and if it has congestion, packets are dropped to relieve the congestion.

RPL routing algorithm that constructs and maintains Destination Oriented Directed Acyclic Graph (DODAGs) [3] through DODAG Information Object (DIO) messages to transmit data from sensors to root over a single path. This information is exchanged as a new type of ICMPv6 message called the RPL Control Message. RPL is a gradient based routing protocol that builds the graph known as Directed Acyclic Graph (DAG) by using a set of metrics/constraints and an objective function.

RPL provides support for a large number of technologies and features that matches all service requirements reviewed in the introduction. One of the key characteristics of RPL is that the protocol is highly flexible and dynamic; it has been designed to operate in harsh environments with low-speed links potentially experiencing high error rates, while generating very low control plane traffic [4]. Routing metrics are used by routing protocols to compute shortest paths. Interior Gateway Protocols (IGPs) such as IS-IS and OSPF use static link metrics.

Presently, several important emerging multicast application such as distributed database systems, radio, television, video conferencing system, distance learning system, are becoming more and more popular. As a result, the portion of multicast traffic on the IP network in almost all organization is increasing rapidly. Therefore, IP network design process should also effectively route multicast traffic in addition to the traditional unicast traffic. A design that concern both unicast and multicast routing is a very difficult problem.

This study proposes a design routing algorithm for WSNs with mixed unicast and multicast traffic called M-RPL. The algorithm is obtained by modifying spanning tree building portion of RPL. M-RPL to construct core spanning tree instead of Prim-Dijkstra algorithm. The performances of networks design by M-RPL are evaluated in term of installation cost and compared with the networks design by original RPL for various traffic demands and networks with different number of nodes. The experimental results show that, in almost all cases, M-RPL gives better performance in term of installation cost.

The reminder of this paper is organized as follows. In Section II survey recent research works that deal with mobility support for RPL. Section III presents a detailed description of M-RPL. The Scilab/NARVAL [5] simulation results related to the comparison of M-RPL against the standard specification of RPL in WSNs are presented in Section IV. Section V concludes the paper and discusses future works. 


\section{RELATED WORK}

There has been some research addressing the problem of extending RPL to support mobility.

F. Melakessou et al. in [6] propose to study the effectiveness of RPL compared to a shortest path algorithm such like the Dijkstra's algorithm. The authors analyzed peer-to-peer communications inside random wireless sensor network topologies. The authors have built a particular simulation environment named Network Analysis and Routing eVALuation (NARVAL). The authors finally performed all paths between each couple of two distinct sensor nodes and compared them to the corresponding shortest paths obtained by the Dijkstra's algorithm. This approach permits to retrieve some statistics on the path extension between RPL and the Dijkstra's algorithm. The authors also analyzed the impact of the sink position and the network size on this path extension.

In [7], the authors studied a Heuristic Load Distribution algorithm (HeLD) based on a braided multipath extension of the standard Routing Protocol for Low power and lossy networks (RPL), which tries to achieve a well-balanced traffic load and simultaneously maximizes the total packet throughput during the network lifetime.

In [8], the authors propose a new routing algorithm called Natural Gradient Routing (NGR), which uses sensed values in a gradient field as a rank, and a single forwarding rule to approach the Sink. With extensive simulation work they compare our proposal against two reference routing algorithms: Flooding, which represents the absence of predefined routes and $\mathrm{AODV}$, a deterministic reactive shortest-path approach.

In [9] presented address this gap and propose Co-RPL as an extension to RPL based on the Corona mechanism to support mobility. They study the impact of node speed, packet transmission rate and number of Directed Acyclic Graphs (DAG) roots on network performance. The simulation results show that Co-RPL decreases packet loss ratio by $45 \%$, average energy consumption by $50 \%$ and end-to-end delay by 2.5 seconds, in comparison with the standard RPL.

More recently, the paper in [10] presented specifies the Multicast Protocol for Low power and Lossy Networks (MPL), which provides IPv6 multicast forwarding in constrained networks. MPL avoids the need to construct or maintain any multicast routing topology, disseminating multicast messages to all MPL Forwarders in an MPL
Domain. By using the Trickle algorithm, MPL requires only small, constant state for each MPL device that initiates disseminations. The Trickle algorithm also allows MPL to be density-aware, allowing the communication rate to scale logarithmically with density.

\section{OVERVIEW OF M-RPL}

In this section, the author describe the M-RPL mechanism for mobile low power and lossy WSNs.

M-RPL starts with node clustering and select the backbone nodes in the same way as RPL. Next, since all multicast transmitters share the same tree to distribute the data, instead of building Prim-Dijkstra tree, the one or more root nodes shortest-path tree is build based on Trickle Multicast (TM) [11], [12] algorithm. The algorithm forms a shortest-path tree with a high rate set of receivers and then successively adds lower rate sets of receivers to the tree and, finally, adds nodes without receiver (zero rate receivers) to the tree. To guide the multicast traffic to flow only through the core shortest-path tree the multicast link weight should be set appropriately. One simple solution is to set the multicast link weight of all links in the shortest-path tree to 1 and that of the others to very large number.

After the author obtained a shortest-path tree, direct links are added and their unicast link weights are determined based on the unicast traffic in exactly the same way as in RPL. The capacity a link on the tree is determined by the sum of its own unicast traffic, the overflow unicast traffic from other routes and the multicast traffic that flow through it.

\section{PERFormanCE EVAluATION}

In this section, the author detail the performance evaluation of M-RPL using NARVAL toolbox, a widely-used and reliable sensor network simulator/emulator under Scilab simulation. In order to evaluate the efficiency of network design calculated by M-RPL algorithm, the author analyze the performances in term of installing cost.

\section{A. Design Example}

A WSNs composes of 100 nodes shown in Fig. 1. The unicast and multicast traffic random generate by Scilab simulation. The installation cost of link with $10 \mathrm{Kbps}$ capacity.

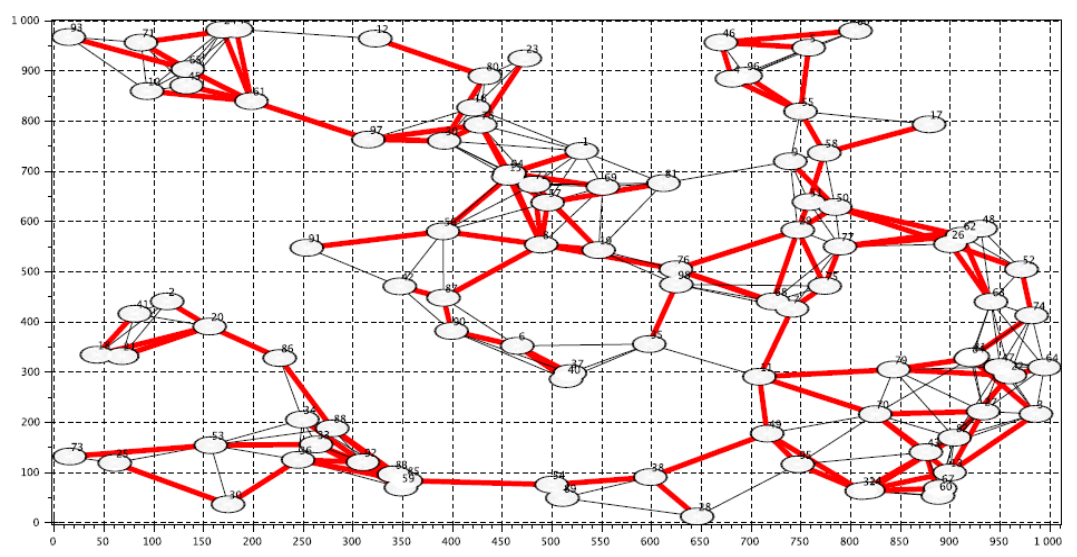

Fig. 1. Final forwarding topology built by M-RPL. 


\section{A. Essential Steps of $M-R P L$}
/*(1) Generate Topology
/*(2) Update of Weight
/*(3) Compute Root Node
/*(4) Build M-RPL Tree
$/ *(5)$ Install Traffic
/*(6) Calculate Link Capacity
$/ *(5)$ Graph Visualization

\section{B. Pseudo Code}

$$
[P, D, R, D A G, D I O]=\mathrm{NL} \_\mathrm{R} \_\mathrm{RPL}(G, S, N)
$$

where arguments are $G$ : Graph, $S$ : Source node (root), $N$ : Storage size, $P$ : Predecessor vector, $D$ : Distance vector, $R$ : Rank vector, $D A G$ : DAG vector and $D I O$ : DIO vector. Shown in Fig. 2.

\section{Network Design Results}

Using Prim-Dijkstra algorithm as in M-RPL. Traffic load on each direction may compose of unicast and multicast traffic. Shown in Fig. 1.

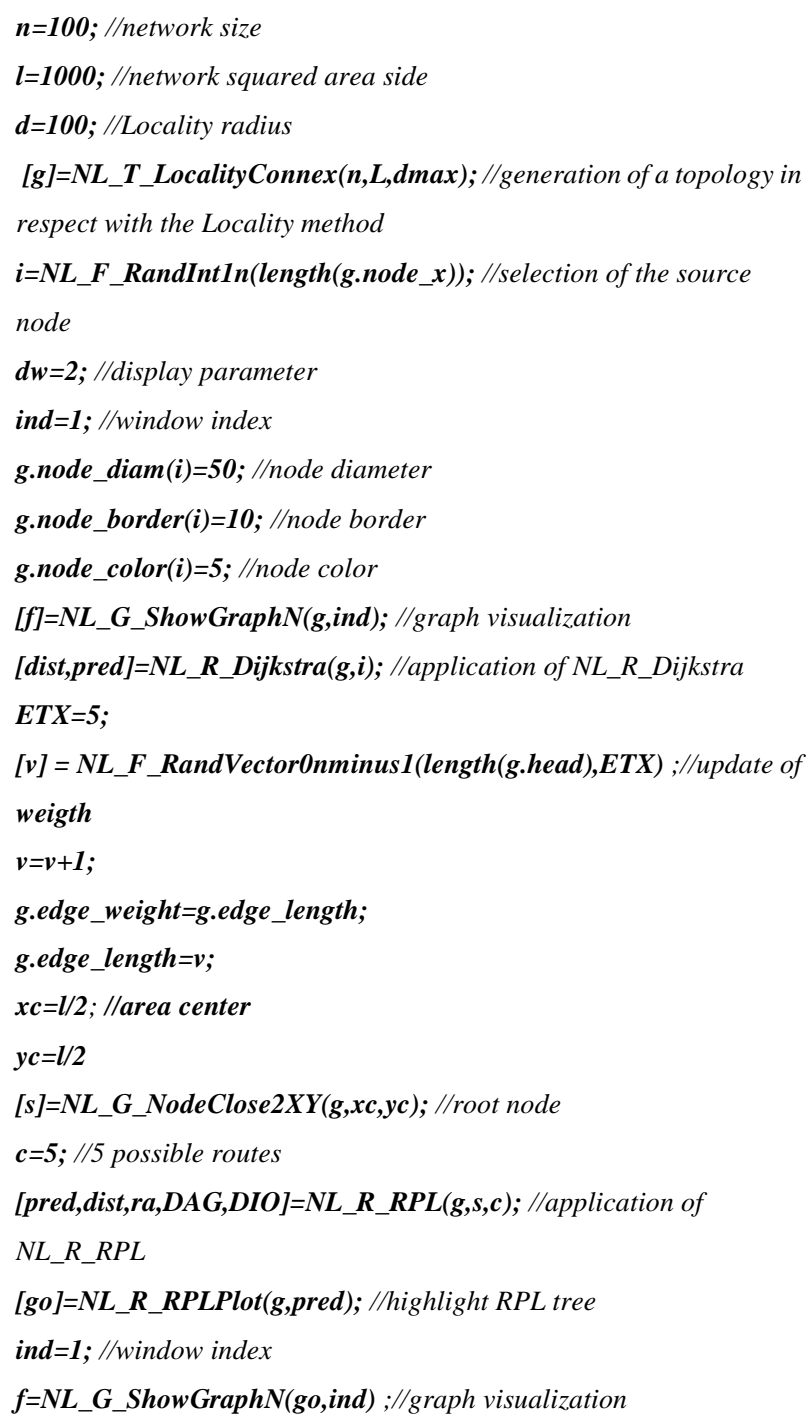

Fig. 2. M-RPL algorithm "Pseudo code".

\section{Installation Cost}

Tables I-V present the installation cost for 100 nodes networks obtained by M-RPL and the total unicast traffic in/out per node of $64,128,256,512$ and $1024 \mathrm{kbps}$. What the author have modified is that all multicast traffics are forced to flow through the spanning tree of the RPL network. And thus the link capacities of the spanning tree are determined by the summation of the unicast and multicast traffic flow through them. The design results obtained by M-RPL. In case, it presented in the tables by order to compare with of network.

TABLE I: INSTALLATION COST OF 100 NODE NETWORK WITH TOTALUNICAST TRAFFIC IN/OUT OF 64 KBPS

\begin{tabular}{lll}
\hline \hline Multicast Traffic & RPL & M-RPL \\
\hline 100 node $\times 128 \mathrm{Kbps}$ & 13430 & 11481 \\
\hline \hline $\begin{array}{c}\text { TABLE II: INSTALLATION COST OF 100 NODE NETWORK WITH } \\
\text { TOTALUNICAST TRAFFIC IN/OUT OF 128 KBPS }\end{array}$ \\
\hline \hline $\begin{array}{l}\text { Multicast Traffic } \\
\text { RPL }\end{array}$ & M-RPL \\
\hline \hline
\end{tabular}

TABLE III: INSTALLATION COST OF 100 NODE NETWORK WITH TOTALUNICAST TRAFFIC IN/OUT OF 256 KBPS

\begin{tabular}{lll}
\hline \hline Multicast Traffic & RPL & M-RPL \\
\hline 100 node $\times 128 \mathrm{Kbps}$ & 15674 & 13962 \\
\hline \hline
\end{tabular}

TABLE IV: INSTALLATION COST OF 100 NODE NETWORK WITH TOTALUNICAST TRAFFIC IN/OUT OF 512 KBPS

\begin{tabular}{lll}
\hline \hline Multicast Traffic & RPL & M-RPL \\
\hline 100 node $\times 128 \mathrm{Kbps}$ & 18385 & 16638 \\
\hline \hline
\end{tabular}

TABLE V: INSTALLATION COST OF 100 NODE NETWORK WITH TOTALUNICAST TRAFFIC IN/OUT OF 1024 KBPS

\begin{tabular}{lll}
\hline \hline Multicast Traffic & RPL & M-RPL \\
\hline 100 node $\times 128 \mathrm{Kbps}$ & 20068 & 18753 \\
\hline \hline
\end{tabular}

\section{CONCLUSION}

This study proposes design routing algorithm for WSNs called M-RPL that support both unicast and multicast traffic simultaneously. However, since multicast traffic model could be employed in many situations and could be managed by various kinds of multicast routing protocols. M-RPL is a modified version of RPL that uses Trickle Multicast (TM) algorithm, rather than Prim-Dijkstra Algorithm, to construct spanning tree. An example of 100 backbone nodes network design is given.

The efficiency of M-RPL is evaluated in term of network installation cost. The installation cost of 100 nodes networks and the total unicast traffic in/out per node of $64,128,256$, 512 and $1024 \mathrm{kbps}$. M-RPL are calculated and compared with that of RPL with various design parameters and various conditions of mixed traffics. It is shown that, all most all cases, M-RPL networks give lowest installation cost. 


\section{REFERENCES}

[1] Q. Le, M. T. Ngo-Quynh, and T. Magedanz, "RPL-based multipath routing protocols for internet of things on wireless sensor networks," in Proc. 2014 International Conference on Advanced Technologies for Communications (ATC), 2014, pp. 424-429.

[2] I. E. Korbi, M. B. Brahim, C. Adjih, and L. A. Saidane, "Mobility enhanced RPL for wireless sensor networks," in Proc. 2012 Third International Conference on the Network of the Future (NOF), 2012, pp. $1-8$.

[3] T. Winter, P. Thubert, A. Brandt, J. Hui, R. Kelsey, P. Levis, K. Pister, R. Struik, J. P. Vasseur, and R. Alexander, "RFC 6550: RPL: IPv6 routing protocol for low-power and lossy networks," Internet Engineering Task Force (IETF) Request For Comments, March 2012.

[4] Cisco White Papers. (January 2014). A Standardized and Flexible IPv6 Architecture for Field Area Networks. [Online]. Available: http://www.cisco.com/c/en/us/products/collateral/routers/1000-seriesconnected-grid-routers/white-paper-c11-730860.pdf

[5] Scilab homepage. [Online]. Available: http://www.scilab.org/

[6] F. Melakessou and T. Engel, "Path extension analysis of peer-to-peer communications in small 6LoWPAN/RPL sensor networks," in Proc. 2013 IEEE 21st International Symposium on Modelling, Analysis \& Simulation of Computer and Telecommunication Systems, 2013, pp. 303-307.

[7] M. N. Moghadam and H. Taheri, "High throughput load balanced multipath routing in homogeneous wireless sensor networks," in Proc. the 22nd Iranian Conference on Electrical Engineering (ICEE 2014), 2014, pp. 1516-1521.

[8] C. Buchhorsts and D. Dujovne, "Natural gradient routing: Sink convergence using data as guide," in Proc. 2013 IEEE Latin-America Conference on Communications (LATINCOM), 2013, pp. 1-6.
[9] O. Gaddour, A. Koubaa, R. Rangarajan, O. Cheikhrouhou, E. Tovar, and M. Abid, "Co-RPL: RPL routing for mobile low power wireless sensor networks using Corona mechanism," in Proc. 2014 9th IEEE International Symposium on Industrial Embedded Systems (SIES), 2014, pp. 200-209.

[10] J. Hui and P. Thubert, "Multicast forwarding using trickle," Internet Draft, Apr. 2011.

[11] P. Levis, N. Patel, D. Culler, and S. Shenker, "Trickle: A selfregulating algorithm for code propagation and maintenance in wireless sensor networks," in Proc. First USENIX/ACM Symposium on Networked Systems Design and Implementation (NSDI), 2004, pp. 15-28.

[12] P. Levis, T. H. Clausen, J. Hui, O. Gnawali, and J. Ko, "The trickle algorithm," RFC 6206, Mar. 2011.

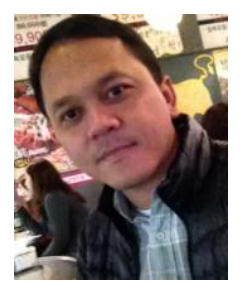

Annop Monsakul received the bachelor degree of industrial technology in power electronics technology from King Mongkut's Institute of Technology North Bangkok, Thailand, in 2000; the graduate diploma of engineering in nuclear technology from Chulalongkorn University, Bangkok, Thailand in 2001; the M.Sc. degree in telecommunications and computer networks from Rangsit University, Pathumthani, Thailand, in 2005; and the Ph.D. degree in information technology, Rangsit University, Pathumthani, Thailand, in 2014. He is currently working as a lecturer in the Faculty of Information of Technology, Thai-Nichi Institute of Technology, his research interests are in the areas of data network topology design algorithm, traffic engineering and the optimization of engineering problems. 



\section{Information Network and Security}


